\title{
COMPLICACIONES NEUROLÓGICAS DEL PACIENTE CON CÁNCER
}

\author{
NEUROLOGIC COMPLICATIONS OF CANCER PATIENT
}

\author{
David López' ', Sandra Valle², Ana Isabel Ferrer³, Juan Coves' , Nieves Galán', \\ Joaquín Gimeno', Isabel Pajares' y Valerie Rodríguez ${ }^{1}$ \\ 1 Servicio de Oncología Médica, Hospital Clínico Universitario Lozano Blesa, Zaragoza \\ 2 Servicio de Hematología y Hemoterapia, Hospital Clínico Universitario Lozano Blesa, Zaragoza \\ 3 Servicio de Oncología Médica, Complejo Hospitalario Universitario de Albacete, Albacete
}

\section{Resumen}

Los avances de las últimas décadas tanto en el diagnóstico como en tratamiento del cáncer han conseguido aumentar la supervivencia, lo que ha conllevado un aumento de las complicaciones neurológicas. Estas complicaciones pueden ser debidas tanto al propio cáncer como al tratamiento y en ocasiones son la primera manifestación de la enfermedad oncológica. Algunas de estas complicaciones son potencialmente reversibles por ello el diagnóstico precoz y su tratamiento correcto pueden mejorar los síntomas neurológicos y la calidad de vida de estos pacientes. En el presente artículo haremos una revisión de las principales complicaciones neurológicas del paciente con cáncer con una orientación diagnóstica y terapéutica.

Palabras clave: Cáncer, complicación, neurología, síndrome, paraneoplásico, quimioterapia, radioterapia.

\section{Abstract}

The advances of recent decades in the diagnosis and treatment of cancer have managed to increase survival, but this has produced an increase in neurological complications. These complications can be caused by cancer and its treatment and sometimes can be the first manifestation of cancer disease. Some of these complications are potentially reversible so that early diagnosis and proper treatment can improve the neurological symptoms and quality of life of these patients. In this article we will review major neurological complications of cancer patients and also do a diagnostic and therapeutic orientation.

Key words: Cancer, complication, neurological, paraneoplastic syndrome, chemotherapy, radiotherapy.

\section{INTRODUCCIÓN}

Los avances que se han producido en las últimas décadas tanto en el diagnóstico como en el tratamiento del cáncer han conseguido aumentar la supervivencia, pero este hecho también ha llevado un aumento de las complicaciones neurológicas, siendo esta una importante causa de consulta en los servicios de urgencias $^{(1)}$. Las complicaciones neurológicas pueden ser debidas tanto al propio cáncer como al

\footnotetext{
Correspondencia

David López Sánchez Servicio de Oncología Médica Hospital Clínico Universitario Lozano Blesa Avenida San Juan Bosco 15 50010 Zaragoza

E-mail: gansinos@hotmail.com
} 
tratamiento y en ocasiones son la primera manifestación de la enfermedad oncológica. El diagnóstico preciso suele ser difícil porque los diferentes trastornos neurológicos pueden presentar signos y síntomas similares lo que puede complicarse además si existen enfermedades neurológicas concomitantes. Debemos considerar que muchas de estas complicaciones son potencialmente reversibles por ello es fundamental el diagnóstico precoz y su tratamiento correcto, para así mejorar los síntomas neurológicos y la calidad de vida de estos pacientes.

En el presente artículo haremos una revisión de las principales complicaciones neurológicas del paciente con cáncer, tanto las debidas al propio tumor por afectación primaria o metastásica, como las debidas a otras causas como encefalopatías metabólicas, accidentes cerebrovasculares, infecciones, síndromes paraneoplásicos y las relacionadas con el tratamiento (quimioterapia y radioterapia). También haremos una orientación diagnóstica y terapéutica.

\section{METÁSTASIS CEREBRALES}

La metástasis intracraneales son la complicación neurológica más común en los pacientes con cáncer, afectando a más de un $25 \%$ de los pacientes con cáncer metástasico y la incidencia está aumentando debido a la mayor capacidad de diagnóstico con las técnicas de nuero-imagen y a un tratamiento más efectivo de la enfermedad sistémica que modifica su historia natural(2).

La metástasis cerebrales son más frecuentes que los tumores primarios cerebrales con una relación 5-10 a $1^{(3)}$ siendo esta afección más frecuente en pacientes con cáncer de pulmón y cáncer de mama, debido a la alta prevalencia de estos tipos de cáncer. Sin embargo el tumor que con más frecuencia metastatiza en el cerebro es la neoplasia trofoblástica gestacional, segui- do del cáncer de pulmón, tumores germinales, hipernefroma, melanoma y cáncer de mama. Dentro del mismo tipo de tumor también hay diferencias en la propensión a metastatizar en el cerebro. Dentro del cáncer de mama, aquellos con receptores hormonales negativos y sobreexpresión del receptor 2 del factor de crecimiento epidérmico humano (HER-2) tienen más tendencia a metastizar en el cerebro ${ }^{(4)}$. Dentro del cáncer del pulmón las metástasis cerebrales son más frecuentes en los tumores de célula pequeña (microcítico) y en los adenocarcinomas que en los carcinomas escamosos.

Las lesiones cerebrales pueden producir síntomas por invasión de tejido cerebral, por compresión de estructuras adyacentes e hipertensión intracraneal. La sintomatología dependerá de la localización, la rapidez de crecimiento y el número de metástasis. El síntoma más frecuente es la cefalea o el cambios de sus características, si esta es habitual en el paciente. Típicamente la cefalea aumenta por la noche debido a la vasodilatación producida por el aumento de la presión parcial de dióxido de carbono. La cefalea también puede ir acompañada de náuseas y vómitos pudiendo ser confundida con una migraña. La disfunción cognitiva suele estar presente, a veces de forma sutil, en forma de cambios del comportamiento, apatía, abulia o somnolencia. Las convulsiones pueden ser el primer síntoma de metástasis cerebrales, siendo especialmente frecuentes en las metástasis del melanoma. También pueden presentar síntomas focales como afasia, amaurosis o pérdidas de fuerza o sensibilidad $^{(5)}$.

El diagnóstico nos lo darán las pruebas de neuro-imagen. La prueba de elección es la resonancia magnética (RM) con gadolinio que es más sensible que la tomografía computarizada en la detección de metástasis cerebrales en fosa posterior, lóbulo temporal o de pequeño tamaño, incluso 
menores a 5 milímetros. La presencia de focalidad neurológica en un paciente con cáncer obliga a la realización prueba de neuroimagen, si no es posible una resonancia magnética, se realizará una tomografía computarizada cerebral (TC) con contraste $^{(6)}$.

Antes de entrar en consideraciones terapéuticas debemos tener en cuenta varios factores. En primer lugar si el tumor primario es conocido; si no lo es y la lesión cerebral por neuroimagen sugiere que se trata de una metástasis habrá que realizar una búsqueda del primario y si este no se localiza interesará la biopsia o resección de la lesión cerebral para estudio anatomopatológico. La estirpe anatomopatológica condiciona el tratamiento, dado que se debe tener en cuenta la radiosensibilidad y la quimiosensibilidad del tumor. En segundo lugar la edad y el estado general del paciente condicionará el tratamiento a seguir, la respuesta al mismo y el pronóstico. Los pacientes con mal estado general tienen un peor pronóstico y una peor respuesta a los tratamientos. Y en último lugar el número, la localización y el tamaño ello determinará su resección y la aplicación de radiocirugía.

Los corticoides son el fármaco de elección para el control sintomático y la disminución del edema, aunque el mecanismo de acción no es bien conocido. El corticoide de elección es la dexametasona por su escaso efecto mineralocorticoide. En el tratamiento específico la quimioterapia tiene escaso papel excepto en los tumores muy quimiosensibles como el cáncer de pulmón célula pequeña o los tumores germinales. Si la lesión primaria es única, el estado general del paciente es bueno y el tumor primario está controlado se puede valorar resección quirúrgica. La radiocirugía tiene utilidad en lesiones irresecables por su localización, como el tronco encefálico, y en aquellas menores de 3 centímetros siempre que el número total de lesiones no sea mayor de tres. En cuanto a la radioterapia holocraneal que es uno de los tratamientos más utilizados, se puede usar como tratamiento único o posterior a la cirugía radiocirugía o quimioterapia $^{(7,, 8)}$.

Debemos recordar que el manejo del paciente siempre es individualizado y que pasar del los tratamientos descritos el pronóstico es malo con una mediana de supervivencia inferior a 6 meses $^{(9)}$.

\section{CARCINOMATOSIS MENÍNGEA}

La carcinomatosis meníngea es definida como la diseminación y crecimiento de las células neoplásicas en el espacio leptomeníngeo. Se presenta aproximadamente en el 5\% de los pacientes con cáncer. Puede presentarse en prácticamente cualquier tipo de cáncer, aunque es más frecuente en pacientes con leucemia linfoblástica aguda y linfoma no Hodgkin de alto grado. En caso de tumores sólidos se presenta frecuentemente en unos estadios avanzados de la enfermedad y asociada en muchos casos a metástasis cerebrales.

En los tumores sólidos que con mayor frecuencia producen carcinomatosis meníngea son el melanoma, el carcinoma de pulmón de células pequeñas y el cáncer de mama. En cuanto los primarios cerebrales el meduloblastoma y el ependimoma se diseminan por el espacio leptomeníngeo.

La alteración más característica es la presencia de síntomas y signos neurológicos múltiples que afectan a diferentes niveles del neuroeje. Los síntomas de presentación más comunes incluyen neuropatías craneales, radiculopatías y síntomas cerebrales generalizados como cefalea, síndrome confusional y crisis epilépiticas.

El diagnóstico se realiza con la demostración de células malignas en el líquido cefaloraquídeo. Este método es altamente 
específico pero poco sensible, pudiendo ser negativo en el $40 \%$ de los casos por lo que si la sospecha es alta es conveniente repetir la toma de muestra. La resonancia magnética con gadolinio también tiene utilidad en el diagnóstico(6).

El tratamiento se fundamenta en la quimioterapia intratecal y ocasionalmente radioterapia si se detectan lesiones focales y sintomáticas. A pesar del tratamiento el pronóstico es ominoso con medianas de supervivencia entre 2 y 3 meses $^{(10,11)}$.

\section{METÁSTASIS ESPINALES Y COMPRESIÓN MEDULAR}

La compresión de la médula por enfermedad metastásica es la afectación de la medula espinal más frecuente. Hasta un $30 \%$ de todos los pacientes con cáncer desarrollan tumores espinales secundarios y la forma más habitual de compresión medular es la metástasis extradural por afectación del hueso vertebral. Una causa menos frecuente son las metástasis intraespinales, en cuyo caso el primario más habitual es el cáncer de pulmón de célula pequeña. La metástasis espinal puede ser la primera forma de presentación aunque lo más frecuente es que cuando aparece el paciente ya tenga diagnosticado una enfermedad oncológica. Aunque puede ocurrir en cualquier tipo de tumor maligno son más frecuentes en el cáncer de mama, próstata y pulmón causando en su conjunto entre un $15-20 \%$ de los $\operatorname{casos}^{(12)}$.

En los pacientes con compresión medular, lo cual se considera una urgencia oncológica, el primer síntoma que el paciente suele presentar es el dolor, a veces de distribución radicular, el cuál puede preceder en varias semanas a la presentación de la focalidad neurológica. La exploración neurológica puede poner de manifiesto alteraciones de la sensibilidad y disminución de la fuerza lo cual, junto al punto doloroso, orienta al nivel de la lesión. La alteración en el control de esfínteres suele ser un síntoma tardío y de mal pronóstico.

La prueba de elección para el diagnóstico para la compresión medular es la resonancia magnética de toda la columna dado que puede haber afectación a varios niveles hasta en un $20 \%$ de los casos. En caso de estar contraindicada se puede realizar una tomografía computarizada de columna.

De la rapidez de la instauración del tratamiento dependerán las secuelas. El primer paso terapéutico consiste en dosis altas de dexametasona para disminuir el edema asociado. El tratamiento específico dependerá del tumor primario. La radioterapia es el tratamiento estándar y el más habitual ${ }^{(13)}$. Se puede valorar quimioterapia en tumores muy quimiosensibles como los tumores germinales y los linfomas de alto grado. La cirugía descompresiva está indicada en casos de primario desconocido, progresión a la irradiación, zonas previamente irradiadas, columna inestable y tumores radioresistentes ${ }^{(14)}$. Otra opción en primarios desconocido es la toma de biopsia previa a la irradiación, dado que si se toma posteriormente a la irradiación la rentabilidad es baja.

En cuanto el pronóstico el grado de recuperación neurológica es inversamente proporcional a la extensión del déficit neurológico en el momento del diagnóstico ${ }^{(15)}$.

\section{ACCIDENTES CEREBROVASCULARES}

Los pacientes con cáncer, al igual que la población general, pueden sufrir accidentes cerebrovasculares, pero debido a la idiosincrasia del paciente con cáncer son más frecuentes. Los accidentes cerebrovasculares pueden ser arteriales o venosos, isquémicos o hemorrágicos o trombo-embólicos.

Las causas del infarto cerebral son las mismas que en los pacientes sin cáncer 
pero se añaden algunos factores específicos asociados al cáncer como el estado de hipercoagulabilidad y el tratamiento, ya que tanto la quimioterapia como la radioterapia pueden predisponer a trombosis arteriales o venosas ${ }^{(16)}$. Dentro de los tratamientos específicos podemos destacar por su importante aumento del riesgo el tamoxifeno ${ }^{(17)}$, utilizado en el cáncer de mama hormono sensible, y los antiangiogénicos, como el bevacizumab ${ }^{(18)}$.

Para la prevención y el tratamiento de las trombosis en los pacientes con cáncer se prefieren las heparinas de bajo peso molecular frente a los anticoagulantes orales por ser igual de eficaces con menor riesgo de sangrado.

Las hemorragias cerebrales de los pacientes con cáncer suelen estar asociadas al sangrado de metástasis y en menor medida a trombocitopenia. En este contexto el uso de corticoides tiene utilidad a diferencia de en la hemorragia cerebral primaria $^{(19)}$.

El manejo del paciente oncológico con accidente cerebrovascular, en cuanto a diagnóstico y tratamiento, debe ser el mismo debe ser el mismo que en el resto de pacientes salvo que el evento esté relacionado con metástasis cerebrales ${ }^{(20,21)}$.

\section{ENCEFALOPATÍAS TÓXICO- METABÓLICAS}

En los pacientes con cáncer las encefalopatías tóxicas y metabólicas son muy frecuentes, potencialmente tratables y reversibles. En esta última característica recae su gran importancia.

El origen suele ser multifactorial y coexistir distintos mecanismos. Dentro de las causas tóxico-metabólicas de síndrome confusional en el paciente con cáncer destacan: fármacos (opioides, anticomiciales, corticoides), hiponatremia, hipercalcemia, hipoxia (por anemia o enfermedad pulmonar), sepsis, hiperamonemia (altera- ción hepática), uremia (renal) y hipercapnia (pulmonar). Se ha de señalar que los opioides, de uso habitual en el paciente con cáncer, pueden producir síndromes en los que principalmente destaca la disminución del nivel de conciencia.

El modo de presentación más habitual es la encefalopatía difusa, en forma de cuadro confusional, aunque en ocasiones pueden asociarse crisis comiciales o focalidad neurológica.

El diagnóstico se fundamenta en la sospecha clínica y en todo paciente oncológico con focalidad neurólogica se debe descartar en primer lugar causa metabólica. La mayoría de ellas se detectan con una historia clínica, una exploración física y una analítica que incluya pruebas de función hepática y renal. El manejo de las encefalopatías tóxico-metabólicas es médico, y debemos tener en cuenta que a menudo el cuadro está desencadenado por el propio tumor por lo que además del tratamiento médico, el tratamiento específico con cirugía, radioterapia y/o quimioterapia ayudarán a resolver el proceso $^{(21)}$.

\section{SÍNDROMES PARANEOPLÁSICOS NEUROLÓGICOS}

Se puede definir los síndromes neurológicos paraneoplásicos como todos aquellos signos y síntomas neurológicos que no son causados por el tumor y sus metástasis o por infección, isquemia o trastornos metabólicos. Suelen aparecer antes del diagnóstico del tumor. Su patogenia se basa en la respuesta inmune contra las células tumorales, que a la vez ataca al sistema nervioso, que presenta antígenos similares a los de las células tumorales. Prueba de ello es la presencia de anticuerpos antineuronales en el suero y líquido cefalorraquídeo de estos pacientes. Estos anticuerpos pueden ser la confirmación del síndrome paraneoplásico y pueden ay- 
udar a diagnosticar a pacientes con síntomas neurológicos no atribuibles al tumor. A continuación mencionaremos los más relevantes.

Degeneración cerebelosa paraneoplásica. Se caracteriza por el rápido desarrollo de disfunción severa pancerebelar. Inicialmente se manifiesta con ataxia de la marcha y posteriormente del tronco y extremidades hasta llegar a ser invalidante. Suele estar asociado al linfoma de Hodking y al cáncer de pulmón célula pequeña y es debido a la gran pérdida de neuronas de Purkinje. El diagnóstico se basa en la clínica y en la presencia de anticuerpos anti-Yo, anti-Ri y anti-Hu en suero y líquido cefalorraquídeo. La resonancia magnética y la tomografía computarizada solo muestran atrofia cerebelosa en etapas avanzadas de la enfermedad.

Síndrome paraneoplásico opsoclonomioclono. El opsoclono es una alteración de la motilidad ocular caracterizada por movimientos conjugados sacádicos de gran amplitud, irregulares y continuos, en todas las direcciones de la mirada. La clínica corresponde a un síndrome subagudo con opsoclono, ataxia de tronco y de la marcha, disfagia, vértigo, diplopía, disartria y oscilopsia. Está asociado a los neuroblastomas y en segundo lugar al cáncer microcítico de pulmón. El diagnóstico es clínico y es habitual la presencia de anticuerpos anti-Ri en suero y líquido cefalorraquídeo.

Degeneración retiniana. Se manifiesta por fotosensibilidad, reducción del campo visual con un escotoma en anillo, pudiendo llegar a la amaurosis. Histológicamente, se observa pérdida de las células ganglionares, con depósito de anticuerpos que también reaccionan con antígenos de las células tumorales. Al observar el fondo de ojo se aprecian depósitos de pigmento y estenosis arteriolar. Se asocia a carcinoma microcítico de pulmón, tumores ginecológicos y melanomas.
Encefalomielitis paraneoplásica. Secaracteriza por la participación de diferentes áreas como el hipocampo, el tallo cerebral inferior, la médula espinal o ganglios de la raíz dorsal. El cuadro clínico refleja la implicación anatómica variable e incluye: los síndromes del tronco cerebral, disfunción autonómica, mielitis y encefalitis límbica. Puede presentarse de forma aislada pero rápidamente afecta a otros niveles del sistema nervioso. La clínica sensitiva suele ser la forma de presentación más frecuente seguida de la encefalitis límbica y cursa con pérdida grave de la memoria reciente, síntomas psiquiátricos como ansiedad, alteración conductual o depresión y síntomas neurológicos como alucinaciones o confusión ${ }^{(22)}$. La neoplasia implicada con más frecuencia es el cáncer microcítico de pulmón. En la mayor parte de los pacientes se detectan anticuerpos anti-Hu y el electroencefalograma muestra enlentecimiento difuso o descargas patológicas a nivel de los lóbulos temporales.

Síndrome paraneoplásico de la persona rígida. El síndrome de la persona rígida (stiff-person) se caracteriza por rigidez de la musculatura axial con espasmos musculares dolorosos. El electomiograma típico muestra actividad muscular continua que es inhibida por la administración de diazepám oral. Se asocia a linfoma de Hodgkin, timoma y cáncer de mama y pulmón. Los pacientes con síndrome de la persona rígida y cáncer de mama presentan en suero y líquido cefalorraquídeo un anticuerpo antianfifisina.

Síndromes de la motoneurona. Algunos síndromes paraneoplásicos presentan rasgos clínicos que recuerdan a la esclerosis lateral amiotrófica. Aunque estudios epidemiológicos han descartado que haya relación entre esta y el cáncer. Son poco frecuentes y asociados fundamentalmente a linfomas.

Neuronopatía sensitiva. Se produce por la afectación de las neuronas del gan- 
glio raquídeo posterior. Debutan con dolor y parestesias, distribución asimétrica, y afectan más a extremidades superiores. Posteriormente se produce entumecimiento y ataxia de extremidades.

Neuropatías sensitivomotoras. Clínica similar a las neuropatías sensitivomotoras de otra etiología y a la polineuropatía desmielinizante inflamatoria crónica. Frecuentemente asociadas al cáncer de pulmón. La neuropatía sensitivomotora subaguda que aparece en alguna ocasión en pacientes con linfoma y leucemia puede ser debida a la infiltración directa de los nervios periféricos.

Síndrome miasteniforme de LambertEaton. Se trata de un problema autoinmune consistente en una inhibición de la liberación presináptica de acetilcolina mediante anticuerpos anticanales del calcio. Es frecuente en el carcinoma microcítico de pulmón dónde se produce en el 3\% de los pacientes. Inicialmente aparece debilidad proximal en extremidades inferiores y astenia, junto a trastornos autonómicos que incluyen visión borrosa con enlentecimiento de reflejos pupilares, impotencia, xerostomía, xeroftalmía y disminución de sudoración. De forma característica, una contracción voluntaria mantenida durante unos segundos provoca un aumento de la fuerza y de los reflejos. El diagnóstico de este síndrome se confirma mediante electromiograma. Es frecuente la presencia anticuerpos en suero contra canales de calcio voltaje dependientes tipo P.

Miastenia gravis. Es un trastorno producido por la acción de anticuerpos frente a receptores de acetilcolina. En los pacientes diagnosticados de timoma puede presentarse hasta en un tercio de los casos. Se trata de un síndrome muy conocido, caracterizado por la debilidad muscular, en especial la debilidad palpebral.

Como hemos comentado en la fisiopatología del síndrome paraneoplásico media la producción autoanticuerpos, por lo que el tratamiento médico del síndrome paraneoplásico consiste en la en tratamiento inmunosupresor con corticoides, inmunoglobulinas y ocasionalmente plasmaféresis. El tratamiento médico suele ser poco efectivo siendo el tratamiento de elección el tratamiento de la enfermedad oncológica subyacente. La presencia de síndromes paraneoplásico confiere un peor prónostico del proceso oncológico de base ${ }^{(23,24)}$.

\section{TOXICIDAD NEUROLÓGICA DE LA RADIOTERAPIA}

La radioterapia, como la mayoría de los tratamientos empleados contra el cáncer, posee una citotoxicidad no específica que afecta tanto a las células tumorales como a las sanas. Pese al desarrollo de nuevos regímenes de aplicación, estas complicaciones continúan siendo un problema importante, sobre todo en aquellos pacientes con mayor esperanza de vida. Los mecanismos por los que la radioterapia produce los diferentes tipos de daño sobre el sistema nervioso no son bien conocidos, aunque parecen implicar fundamentalmente procesos de daño vascular y desmielinización tanto por daño directo así como por la inflamación y alteración de la permeabilidad que provoca sobre la barrera hematoencefálica.

Tradicionalmente se ha clasificado en toxicidad aguda (aparecen en los primeros 30días), subaguda y crónica o diferidas tardías (a partir de los 3 meses de la radiación). Habitualmente los efectos agudos y subagudos suelen ser más leves y reversibles frente los crónicos que son irreversibles.

\section{Efectos agudos}

La encefalopatía aguda suele aparecer los primeros días (fundamentalmente en las primeras 24 horas) sobre todo en pacientes con fraccionamientos superiores a 
3 greys. Consiste en un cuadro transitorio de cefalea intensa, somnolencia, náuseas, vómitos, empeoramiento de los déficit neurológicos previos y fiebre. Suele ser más intenso con la primera dosis de radiación y luego ir cediendo progresivamente. La causa parece ser el edema cerebral secundario a la alteración de la permeabilidad de la barrera hematoencefálica. Para prevenirlo se administra a los pacientes dexametasona.

El deterioro focal neurológico está a caballo entre toxicidad aguda y crónica. Se observa entre semanas y meses después de finalizada la radioterapia. Suele constatarse un deterioro focal transitorio con cefalea o somnolencia. Se asocia a edema peritumoral y desmilinización. En la mayoría de los casos es reversible.

\section{Efectos crónicos}

La radionecrosis, es una complicación grave que suele producirse 1-2 años tras el inicio del tratamiento, aunque puede aparecer desde meses hasta décadas tras la radiación. Esta causada por un daño en el endotelio vascular que se traduce en una necrosis fibrinoide de las pequeñas arterias, causando una necrosis coagulativa y desmielinazación del parénquima cerebral circundante. Ello provoca alteraciones neurológicas dependientes del área afectada. La RM muestra una lesión con efecto masa, necrosis, edema perilesional y captación de contraste, en ocasiones con un patrón típico de "pompas de jabón" o "queso suizo". Puede ser confundido con recurrencia de la enfermedad oncológica. El tratamiento se fundamenta en corticoides para reducir el edema.

La leucoencefalopatía consiste en alteraciones de la sustancia blanca objetivables en resonancia magnética y la clínica es inespecífica incluyendo el deterioro cognitivo. A menudo es difícil atribuir el deterioro cognitivo a la radioterapia dado que subyacen otros factores como la propia progresión tumoral.

La radioterapia sirve para tratar tumores, pero también los produce al tratarse de radiaciones ionizantes. Se ha descrito tras la radioterapia craneal un aumento en la incidencia de meningiomas, gliomas y schwannomas $^{(25)}$. Estos tumores se caracterizan por su agresividad tanto clínica como histológica, mostrando en la mayoría de los casos una localización múltiple.

Finalmente la radioterapia también puede tener efecto sobre las vías ópticas, produciendo cataratas, neuropatía del nervio óptico y retinopatía ${ }^{(26,27)}$.

\section{TOXICIDAD NEUROLÓGICA DE LA QUIMIOTERAPIA}

La frecuencia de las complicaciones neurológicas en los pacientes con cáncer tratados con quimioterapia ha aumentado considerablemente en los últimos años, debido principalmente a la mayor agresividad e intensidad de los tratamientos y al aumento de la supervivencia de los pacientes, que ha llevado a la aparición de efectos tóxicos tardíos. A continuación mencionaremos los síndromes más comunes y los citostáticos responsables.

Neuropatía periférica. Es la alteración neurológica más frecuente, relacionada con las sales de platino (cisplatino y oxaliplatino) y los taxanos (vinorelvina, vincristina, paclitaxel y docetaxel).Aparece por alteraciones en las fibras de conducción nerviosa, lo que lleva a la pérdida de sensibilidad generalmente en las extremidades (característicamente distribución en guante y calcetín). Clínicamente se describe como parestesias y disestesias que pueden resultar incapacitantes. Aparecen a las semanas del tratamiento, suelen ser dosis dependientes y presentan una mejoría lenta y progresiva tras el cese de la quimioterapia aunque no suele ser completa. El oxaliplatino, provoca de manera frecuente y ca- 
racterística una toxicidad aguda muy frecuente en forma de alteraciones sensitivas en zona perioral, distal y en un pequeño porcentaje de pacientes también alteraciones faringeolaríngeas. Es especialmente relevante que es una toxicidad agravada por el contacto con superficies frías y la ingesta de alimentos y bebidas frías ${ }^{(28)}$.

Encefalopatía aguda. Se inicia durante el tratamiento, y consiste en confusión progresiva asociada a alucinaciones, afasia, letargia, somnolencia y ocasionalmente convulsiones y coma. Se resuelve habitualmente entre 10-14 días después del cese del tratamiento, aunque ocasionalmente pueden quedar daños irreversibles. También puede provocar encefalopatía crónica que se desarrolla a partir de los 2 meses después del tratamiento. Los citostáticos relacionados son citarabina, metrotexate, ifosfamida, carmustina y cisplatino. Entre ellos es especialmente característica la encefalopatía por ifosfamida que puede producir encefalopatía en un $12 \%$ de los pacientes, el antídoto en este caso es el azul de metileno que compensa la toxicidad mitocondrial los metabolitos de la ifosfamida ${ }^{(29)}$. También puede ser útil la tiamina intravenosa ${ }^{(30)}$.

Toxicidad cerebelosa. Aparecen síntomas de daño cerebeloso: ataxia, dismetría, disartria y nistagmo. Su inicio suele ser agudo (durante el tratamiento) y pueden quedar daños irreversibles. Se ha relacionado con el 5-fluoracilo y la citarabina.

Mielopatías. Relacionadas con la administración intratecal de citarabina o metrotexate. Semiológicamente puede aparecer urinaria o fecal y alteraciones sensitivas $\mathrm{o}$ motoras a un determinado nivel.

Deterioro cognitivo. El deterioro cognitivo en los pacientes que han recibido quimioterapia es muy difícil de valorar, dado la gran cantidad de factores que influyen. Ha sido estudiado en pacientes con quimioterapia adyuvante dado que implica mayor supervivencia y se han detectado déficit neuropsicológicos en los pacientes que recibieron la quimioterapia adyuvante que no se ven en los pacientes que recibieron terapia local ni en controles sanos. De todos modos estos cambios cognitivos no se relacionan con deterioro en la calidad de vida de estos pacientes ${ }^{(31,32)}$.

Salvo el caso de la ifosfamida que ya hemos comentado no existe tratamiento específico para esta toxicidad, de forma que el objetivo del tratamiento es el alivio sintomático. Para ello se utilizan analgésicos, antidepresivos (amitriptilina) y anticonvulsionantes (gabapentina y pregabalina). Con fines preventivos se han utilizado neuroprotectores (infusiones de calcio $y$ magnesio, glutamina, vaminofostina) aunque no hay datos concluyentes sobre su eficacia $^{(28,33,34)}$.

\section{DELIRIO}

El delirio es un cambio agudo en la función cognitiva caracterizado por confusión, desorientación, disminución de la capacidad de atención y percepción errónea. La etiología suele ser multifactorial, pudiendo ser la causa cualquiera de los procesos descritos en este artículo por sí solos pero, como hemos comentado, es habitual que haya más de una causa asociada. Las causas más frecuentes de delirio en los pacientes con cáncer son las metabólicas seguidas de las metástasis. En las causas metabólicas suele coexistir más de una alteración, por ejemplo fiebre, anemia, alteraciones iónicas etc. El delirio pone de manifiesto una situación clínica de gravedad y obliga a descartar una causa orgánica subyacente.

Podemos distinguir dos tipos de delirio, el delirio hiperactivo, el cual es fácil de reconocer y el delirio hipoactivo que es más difícil de identificar y puede ser confundido con depresión. El tratamiento debe ir dirigido a la causa desencadenante y sintomáticamente se pude utilizar 
el metilfenidato en el delirio hipoactivo y neurolépticos en el delirio hiperactivo, en este caso el haloperidol es el neuroléptico de elección ${ }^{(21,35,36)}$.

\section{CONCLUSIONES}

Como hemos visto las complicaciones neurológicas de los pacientes con cáncer son secundarias a varios procesos, algunos aún no bien conocidos. Aunque nosotros por motivos didácticos hemos desglosado las diferentes causas no debemos olvidar que en el paciente pueden coexistir varios procesos, (un paciente con metástasis cerebrales será sometido a tratamiento radioterápico y quimioterápico, tomará corticoides y puede padecer alteraciones metabólicas secundarias a trastornos iónicos e infecciones). Esta complejidad hace difícil en ocasiones el correcto diagnóstico, el cual es muy importante porque muchos pacientes con cáncer tienen complicaciones neurológicas que son potencialmente reversibles con el adecuado tratamiento mejorando así su calidad de vida y su supervivencia.

\section{REFERENCIAS BIBLIOGRÁFICAS}

1. Ferrer A, López D, Vidal $M$, Tobeña $M$, Serrano S, Pajares I, E. et al. Evaluation of neurological symptoms in oncologic patients at the emergency department. J Clin Oncol (Meeting Abstracts) 2009; 27;15S: e20728.

2. Gavrilovic IT, Posner JB. Brain metastases: Epidemiology and pathophysiology. J Neurooncol 2005; 75: 5-14. Doi: 10.1007/ s11060-004-8093-6.

3. Wen PY, Schiff D, Kesari S, Drappatz J, Gigas DC, Doherty L, et al. Medical management of patients with brain tumors. J Neurooncol 2006; 80: 313-32. Doi: 10.1007/s11060006-9193-2.

4. Hines SL, Vallow LA, Tan WW, McNeil RB, Perez EA, Jain A. Clinical outcomes after a diagnosis of brain metastases in patients with estrogen- and/or human epidermal growth factor receptor 2-positive versus triplenegative breast cancer. Ann Oncol 2008; 19: 1561-65. Doi: 10.1093/annonc/mdn283.

5. Steeg PS. Tumor metastasis: mechanistic insights and clinical challenges. Nat Med 2006; 12: 895-904. Doi: 10.1038/nm1469

6. Cha S. Neuroimaging in neuro-oncology. Neurotherapeutics 2009;6:465-77. Doi: 10.1016/j.nurt.2009.05.002.

7. Mintz AH, Kestle J, Rathbone MP, Gaspar L, Hugenholtz H, Fisher B, et al. A randomized trial to assess the efficacy of surgery in addition to radiotherapy in patients with a single cerebral metastasis. Cancer 1996; 78: 1470-76. Doi: 10.1002/(SICI)1097$0142(19961001) 78: 7<1470:$ :AID CNCR14>3.0.CO;2-X.

8. Rades D, Kueter JD, Pluemer A, Veninga T, Schild SE. A matched-pair analysis comparing whole-brain radiotherapy plus stereotactic radiosurgery versus surgery plus whole-brain radiotherapy and a boost to the metastatic site for one or two brain metastases. Int J Radiat Oncol Biol Phys 2009; 73: 1077-81. Doi: 10.1016/j. ijrobp.2008.05.035.

9. Kamar FG, Posner JB. Brain metastases. Semin Neurol 2010; 30:217-35. Doi: 10.1055/s-0030-1255225.

10. Chamberlain MC. Leptomeningeal metastasis. Curr Opin Oncol 2010; 22:62735. Doi: 10.1097/CCO.0b013e32833de986.

11. O'Meara WP, Borkar SA, Stambuk HE, Lymberis SC. Leptomeningeal metastasis. Curr Probl Cancer 2007; 31: 367-424. Doi: 10.1016/S0147-0272(07)00067-0.

12. Taylor JW, Schiff D. Metastatic epidural spinal cord compression. Semin Neurol. 2010; 30:245-53. Doi: 10.1055/s-00301255221.

13. Rades D, Abrahm JL.The role of radiotherapy for metastatic epidural spinal cord compression. Nat Rev Clin Oncol 2010; 7: 590-8. Doi: 10. 1038\%2Fnrclinonc.2010.137. 
14. Quraishi NA, Gokaslan ZL, Boriani S. The surgical management of metastatic epidural compression of the spinal cord. J Bone Joint Surg Br 2010;92:1054-60. Doi: 10.1302\%2F0301-620X.92B8.22296.

15. Eleraky M, Papanastassiou I, Vrionis FD. Management of metastatic spine disease. Curr Opin Support Palliat Care 2010; 4:1828. Doi: 10.1097/SPC.0b013e32833d2fdd.

16. Cestari DM, Weine DM, Panageas KS, Segal AZ, DeAngelis LM.Stroke in patients with cancer: incidence and etiology. Neurology 2004; 62: 2025-30.

17. Bushnell CD, Goldstein LB. Risk of ischemic stroke with tamoxifen treatment for breast cancer: A meta-analysis. Neurology 2004; 63:1230-3.

18. Besse B, Lasserre SF, Compton P, Huang J, Augustus $S$, Rohr UP. Bevacizumab safety in patients with central nervous system metastases. Clin Cancer Res 2010; 16: 269-78. Doi: 10.1158/1078-0432.CCR-092439.

19. Poungvarin N, Bhoopat W, Viriyavejakul A, Rodprasert P, Buranasiri P, Sukondhabhant $\mathrm{S}$, et al. Effects of dexamethasone in primary supratentorial intracerebral hemorrhage. N Engl J Med 1987; 316: 1229-33. Doi: 10.1056/NEJM198705143162001.

20. Rogers LR. Cerebrovascular complications in cancer patients. Neurol Clin 2003; 21: 167-92. Doi: 10.1016/S07338619(02)00066-X.

21. Khasraw M, Posner JB. Neurological complications of systemic cancer. Lancet Neurol 2010; 9:1214-27. Doi: 10.1016/ S1474-4422(10)70220-9.

22. Tuzun E, Dalmau J. Limbic encephalitis and variants: classification, diagnosis and treatment. Neurologist 2007; 13: 261-71. Doi: 10.1097/NRL.0b013e31813e34a5.

23. Tres A, Lambea J, Millastre E. Síndromes paraneoplásicos. En: Cortés-Funes $H_{\text {., }}$ editor. Tratado de Oncología. Tomo II. Barcelona: Permanyer, 2009. p. 789-93.

24. Darnell RB, Posner JB. Paraneoplastic syndromes affecting the nervous system.
Semin Oncol 2006; 33: 270-98. Doi: 10.1053/j.seminoncol.2006.03.008.

25. Prasad G, Haas-Kogan DA. Radiationinduced gliomas. Expert Rev Neurother. 2009;9:1511-7. Doi: 10.1586/ern.09.98.

26. Dropcho EJ. Neurotoxicity of radiation therapy. Neurol Clin. 2010; 28:217-34. Doi: 10.1016/j.ncl.2009.09.008.

27. Young DF, Posner JB, Chu F, Nisce L. Rapid-course radiation therapy of cerebral metastases: Results and complications. Cancer 1974; 34: 1069-76. Doi: 10.1002/1097-0142(197410)34:4<1069:: AID-CNCR2820340416>3.0.CO;2-4.

28. Kaley TJ, Deangelis LM. Therapy of chemotherapy-induced peripheral neuropathy. Br J Haematol 2009; 145: 3-14. Doi: 10.1111/j.1365-2141.2008.07558.x.

29. Sweiss KI, Beri R, Shord SS. Encephalopathy after high-dose ifosfamide: A retrospective cohort study and review of the literature. Drug Saf 2008; 31: 989-96.

30. Lombardi G, Zustovich F, Nicoletto MO, Donach M, Pastorelli D. Important role of thiamine in preventing ifosfamideinduced encephalopathy. J Oncol Pharm Pract 2009; 12: 237-39. Doi: 10.1177/1078155209342134.

31. Vardy J, Rourke S, Tannock IF. Evaluation of cognitive function associated with chemotherapy: A review of published studies and recommendations for future research. J Clin Oncol 2007; 2455-63. Doi: 10.1200/JCO.2006.08.1604.

32. Dietrich J, Monje M, Wefel J, Meyers C. Clinical patterns and biological correlates of cognitive dysfunction associated with cancer therapy. Oncologist. 2008; 13:1285-95. Doi: 10.1634/ theoncologist.2008-0130.

33. Dropcho EJ. Neurotoxicity of cancer chemotherapy. Semin Neurol 2010; 30:273-86.

34. Grothey A, Nikcevich DA, Sloan JA, Kugler JW, Silberstein PT, Dentchev T, et al. Intravenous calcium and magnesium for oxaliplatin-induced sensory neurotoxicity 
in adjuvant colon cancer: NCCTG N04C7. J Clin Oncol. 2011; 29:421-7. Doi: 10.1200/ JCO.2010.31.5911.

35. Doriath V, Paesmans M, Catteau G, Hildebrand J. Acute confusion in patients with systemic cancer. J Neurooncol 2007;
83: 285-89. Doi: 10.1007/s11060-0069319-6.

36. Bush SH, Bruera E. The assessment and management of delirium in cancer patients. Oncologist 2009;14: 1039-49. Doi: 10.1634/theoncologist. 2009-0122. 